\title{
INSTRUMENTATION \& TECHNIQUES An electronic device for detecting activity in caged rodents
}

\author{
ROGER M. TARPY and ROBERT J. MURCEK \\ Bucknell University, Lewisburg, Pennsylvania
}

\begin{abstract}
An electronic device for detecting movement in small animals is described. The principle involves assessing changes in resistance between pairs of grid bars in a cage. The device is inexpensive, sensitive to movement, easy to calibrate and install, reliable across different activity arenas, and indifferent to animal size.
\end{abstract}

It is clear that activity has long been an important behavioral measure in experimental psychology. Many investigators have used "general" or "spontaneous" locomotion as an index of, for example, hunger and thirst, sexual receptivity in females, diurnal cycle, exploratory drive, fear, incentive motivation, reactivity to drug injections, and the like. Of course, one can recognize the utility of using activity as an index of these psychological processes regardless of the exact topography that such changes in body position entail. That is, rather than a "state" or condition in itself, activity is more properly viewed as a cluster of specific behaviors, which, depending upon the measuring device, may or may not reflect the presence of other meaningful psychological conditions, such as hunger. Despite the pervasive use of activity as a measure, however, it is difficult to find inexpensive devices that conveniently, reliably, and consistently measure various activity behaviors.

The most common type of device is a mechanical arrangement in which a cage is mounted, often on springs, in such a way that activity causes a switch closure (e.g., Campbell, 1954), a change in the inductance of a coil (e.g., Hoffman \& Fleshler, 1964; Zeier \& Tschannen, 1968), or a piezoelectric effect in a crystal (e.g., Beach, 1970; Cunningham, Crowell, Eaton, \& Brown, 1973; Davis, 1970). Stabilimeters such as these are commercially available, but they are expensive and difficult to calibrate.

Other sorts of devices have made use of electronic circuits (e.g., Morgret \& Albee, 1974; Porter, Hudy, \& Furber, 1979; Van-Toller \& de Sa, 1968). Many of these are somewhat better than mechanical systems, but they usually require special cage floors (e.g., conducting plates rather than grids), and often record only very gross changes in body position. Photocell arrangements are satisfactory, but only when the activity arena is equipped with a sufficient number of independent photobeam circuits to detect small body movements. This makes these systems awkward to

Reprints are available from Roger M. Tarpy, Department of Psychology, Bucknell University, Lewisburg, PA 17837. install in some situations. Commercially available systems of this type are relatively expensive. Inexpensive ultrasonic movement detectors (e.g., Akaka \& Houck, 1980) are available, but these also may be difficult to use in certain situations. More important, calibration can be a problem, since activity, as measured by these devices, varies with the body size of the animal.

Finally, many investigators have used exercise wheels to study "spontaneous" activity. Although the amount of running in a wheel may provide a good index of some psychological states, wheel running is generally considered to be substantially idiosyncratic (e.g., Tapp, Zimmerman, \& D'Encarnacao, 1968; Treichler \& Hall, 1962) and, thus, an unsuitable alternative to the stabilimeter arrangements noted above.

In summary, despite the availability of many sorts of activity measuring devices, no single type to date appears to combine features such as low cost, reliability, easy calibration, easy installation, sensitivity, easy interfacing with other units, and so forth. This paper describes an electronic activity device with reduced cage demands that is very sensitive to the slightest movement, is indifferent to animal size, can be accurately calibrated, and is consistent across multiple activity boxes.

\section{THEORY OF OPERATION}

When an animal is placed on a set of suitably interconnected grid bars, the cage system becomes a variable resistor. Any change in the animal's position, whether due to walking or merely grooming, causes at least a momentary change in the cage system resistance. Activity detection is achieved by measuring the changes in resistance. The problem, of course, with any sort of activity measuring device is to establish criteria for changes in the system that constitute meaningful activity patterns on the part of the animal, and to convert these changes into appropriate signals that can be used by the data-recording devices.

Figure 1 shows the major components of the ac- 


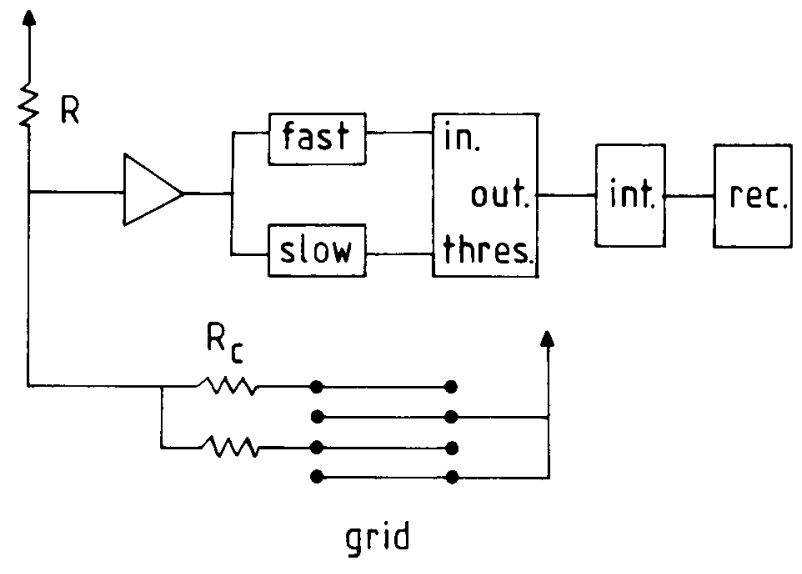

Figure 1. Block diagram of the activity system. The fast and slow filters feed into the window comparator, the output of which drives the recording devices (REC.) via the interface (INT.). Resistors $R$ and $R c$ form a voltage divider, where $R c$ is the effective resistance of the cage system. Four of the 16 bars are shown.

tivity measuring system. The grid floor on a standard Skinner box is connected as shown in Figure 1. Resistor $\mathbf{R}$ and the cage resistance $\mathbf{R c}$ form a voltage divider, producing a voltage that changes with activity. After buffering, the voltage travels two different paths to become two inputs to a window comparator: one through a filter and the other direct. The filtered version of the voltage, which represents the average voltage over a small preceding interval, is used to program the upper and lower thresholds of the window comparator. The unfiltered version is then "watched" by the window comparator, which signals when the present value of the voltage is more than a certain percentage removed from the average value. Because the comparator's output signal can be both very short (less than $10 \mathrm{msec}$ ) and very long (as long as an animal might continue to move), an interface is required between the comparator and the recording equipment (e.g., computer, electromechanical counters, etc.). The interface processes the comparator output and produces a signal that reverses state every $100 \mathrm{msec}$ if activity was detected in the preceding $100-\mathrm{msec}$ interval. With a minimum time of 100 msec between changes, it is therefore well suited to change-of-state-oriented computer software.

\section{CIRCUIT DESCRIPTION}

\section{Cage}

A value of $4.7 \mathrm{M}$ Q was chosen for the grid resistors (see Figure 1). This value is large enough to ensure that no more than $1-\mu \mathrm{A}$ can flow through any one grid. Because the value of these resistors is so large relative to that of a rat (e.g., see Campbell \& Teghtsoonian, 1958), changes in the skin resistance of an animal exert virtually no effect on the system. These resistors are mounted directly on the grid bars, with their common connection brought to the detector circuit via a single-conductor shielded wire.

\section{Detector}

Figure 2 is a schematic diagram of the circuit. The circuit begins with a $1.5-\mathrm{M} \Omega$ resistor to $+5 \mathrm{~V}$. This resistor is the upper resistor in the voltage divider; the cage system is the lower resistor. An FET-input buffer drives two voltage divider/filter networks without loading down the divider.

The network R5, R6, R7, and C2 dynamically sets the thresholds for a pair of comparators, forming an adaptive window comparator. The opencollector comparator outputs are "wire OR'd"; that is, if either comparator output goes low, a lowactive signal results. When the input is not changing, both comparator outputs will be high. When the input signal is changing, $\mathrm{C1}$ and $\mathrm{C} 2$ come into play. Changes in the input of window-comparator-threshold (WCT) magnitude that occur in less than 20 microsec (i.e., noise) are removed by both $\mathrm{C} 1$ and $\mathrm{C} 2$, and are not "seen" by the window comparator. Changes of WCT magnitude that occur over an interval longer than $68 \mathrm{msec}$ are followed closely by C2; since the WCTs move with the change, both comparators' outputs remain high. WCT magnitude changes occurring in 0.2 to $6.8 \mathrm{msec}$, however, will not be removed by $\mathrm{C} 1$ and cannot be followed closely by $\mathrm{C} 2$. This type of change causes either the upper or lower comparator's output to pull low, depending upon whether the change is positive- or negativegoing, respectively.

The relative level of the WCT is set by R6. For the described voltage-divider scheme, the smallest changes that must be detected occur when all but one of the bars are shorted to ground continuously by the animal and activity is causing occasional shorting

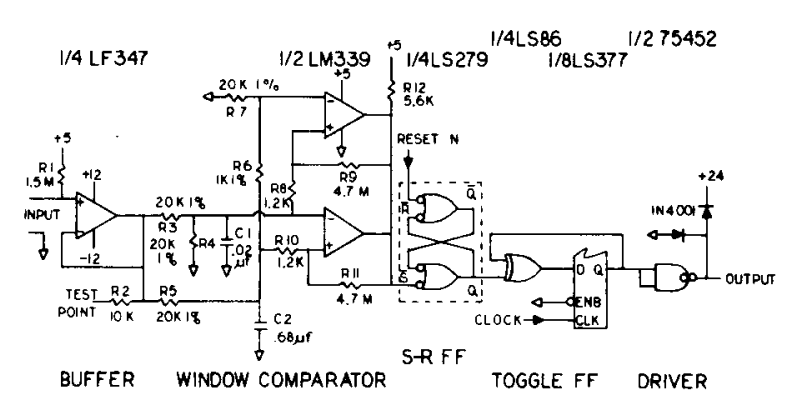

Figure 2. Activity detector channel. The junction of the cage resistors is brought to the INPUT using shielded wire; the OUTPUT is low-active. Good analog design practices, including bypassing of supply voltages and separation of analog, digital, and driver grounds, should be followed. The CLK and ENB inputs to the LS377 are common to all eight of its D fip-flops. 
of the last bar. Assuming a cage with eight sensing bars, nominal values for 1.5 - and 4.7-M $\Omega$ resistors, a 5-V dc supply, and negligible animal resistance, worst-case activity will result in a voltage-divider output fluctuating between 1.41 and $1.55 \mathrm{~V}$. This output is divided in half by $R 3$ and R4, resulting in a window comparator input that fluctuates between 0.71 and $0.78 \mathrm{~V}$, a change of $70 \mathrm{mV}$. Using the customary $50 \%$ threshold, R6 should thus be about $1 \mathrm{k} \Omega$.

\section{Interface}

The remaining channel circuitry is an interface between the window comparator output and the recording equipment. When the output of a comparator goes low, that is, signals activity, an SR flip-flop is set to ensure that a short (less than $10 \mathrm{msec}$ ) pulse will not be lost. The SR flip-flop output passes to the output driver via a "toggle" flip-flop formed by the combination of an exclusive-OR gate and a D-type flip-flop. The toggle flip-flop is clocked at $100-\mathrm{msec}$ intervals. Shortly after the clock pulse, the SR flipflop is reset to begin the watch over the next 100 msec interval. The driver produces a low-active output that is compatible with standard $24-\mathrm{V}$ dc electromechanical equipment.

\section{Timer}

The timing generator (see Figure 3) uses a dual timer to produce the clock and reset signals required by the interface. The first timer section is connected in the standard low-duty-cycle configuration and produces a 6-msec high-active clock pulse every $100 \mathrm{msec}$ (adjustable). The second timer section is slaved to the first section to produce 4.5 -msec lowactive reset pulses beginning $1.5 \mathrm{msec}$ after the leading edge (i.e., positive-going) of each clock pulse. During a clock pulse, the slaved timer's output becomes in-
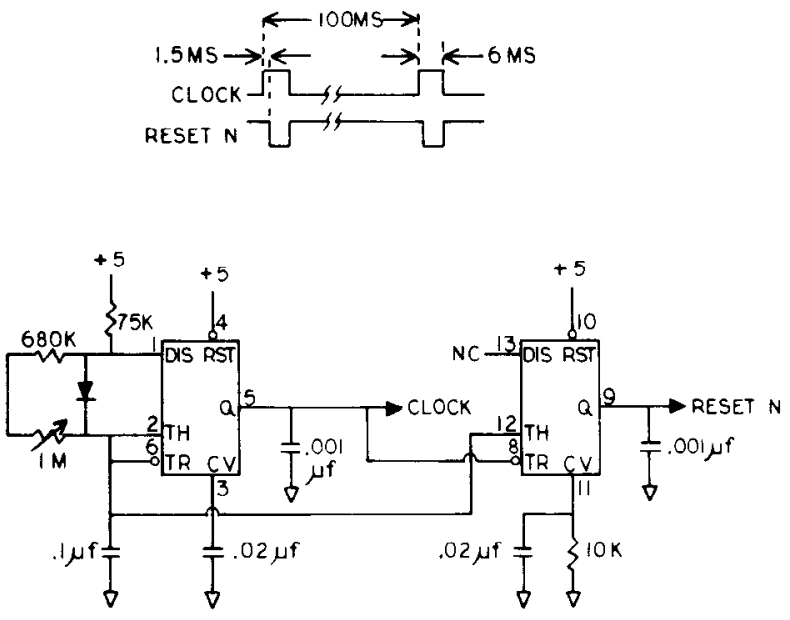

NE 556

Figure 3. Timing generator. For the described application, the potentiometer was adjusted for precise 100 -msec intervals. The outputs can drive at least 100 detection channels.

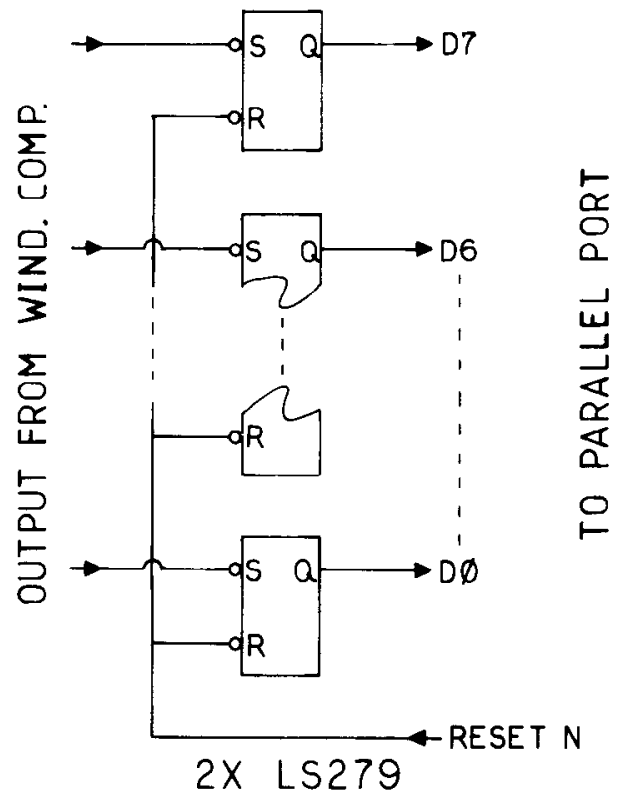

Figure 4. Parallel port interface. RESET $N$ must be $a$ lowactive pulse, preferably less than 2 msec in duration. The handshake lines for most ports can be configured to provide this signal.

active if the voltage at its threshold input exceeds the threshold reference level. The threshold input is obtained from the first timer's timing capacitor, and the threshold reference level is moved from the standard $.67 \mathrm{Vcc}$ to $.50 \mathrm{Vcc}$ by a $10-\mathrm{k} \Omega$ resistor to ground on the control voltage pin. The result is that the pseudotiming cycle of the slaved timer ends a calculable time after a clock pulse begins, initiating a reset pulse. The reset pulse is terminated by the return of the clock signal to the low state.

\section{Other Interfaces}

For interfacing computer systems, a superior interface results from the omission of the toggle flip-flops and the associated timing generator, substituting instead an arrangement as shown in Figure 4.

With this scheme, the computer itself resets the SR flip-flops after reading data, thus reducing the sampling errors. The "Reset $N$ " signal can be transmitted in many ways. The most straightforward would be to use 1 bit of a parallel output port. Alternatively, many microcomputers use interface port chips that can be software-programmed to produce a pulse on a separate control line when the input port is read. Making more extensive use of the computer, the window comparator function could be implemented in software by adding an analog-to-digital converter. It would probably be necessary to resort to assembly language to get a sufficiently short $(10-\mathrm{msec})$ sampling interval when multiple channels are required, however.

To drive electromechanical counters, the arrangement shown in Figure 5 is suggested. This circuit produces a 100 -msec pulse followed by a 100 -msec inactive period for each detected bout of activity. 


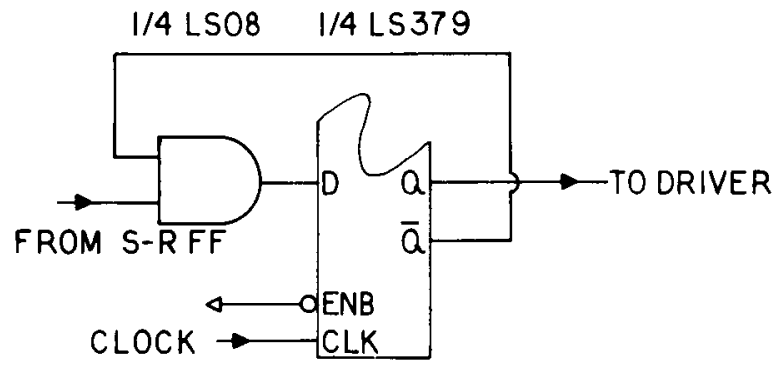

Figure 5. Electromechanical counter compatibility modification. Note change from an octal to a quad D flip-flop.

\section{QUALIFICATIONS}

Several features of the design were included specifically to ensure repeatability, channel-to-channel consistency, and time and environmental stability. First, precision resistors are used to eliminate adjustments to and drifting of sensitivity-related circuits. Where $1 \%$ resistors are specified, they must be used. Second, when more than one device is used, the outputs are conditioned using a common clock signal to ensure that all channels will have similarly timed outputs.

Changes in the cage system voltage divider affect sensitivity; if the resistor values, source voltage, or number of bars is changed, R6 must be recalculated. Because noise and device offsets become more significant as the window becomes smaller, the circuit will probably not be reliable for configurations using more than 10 pairs of sensing bars. Substitution of pin-compatible CMOS comparators for the LM 339s eases this limitation somewhat.

This device has some limitations. First, it may be unsuitable for some activity arenas, particularly those without a conventional grid-bar arrangement. Perhaps a more severe limitation is that the grid bars cannot be used simultaneously for electric shock stimuli unless the grids are remote-switched by a multiple-pole relay located near the cage. This limitation is, of course, shared by virtually any electronic activity measuring device. (We have not tested this scheme. Noise could be a problem unless the wiring associated with the bar resistors is shielded.)

It is best if the cage is metal and is connected to a good ground, that is, a copper water system. The sensing cable shield should not be used for this purpose.

There is an advantage in using this device with a conventional grid floor: The leakage resistance path from a sensing bar to any other bar or ground is relatively long, and this greatly reduces the probability of fouling by excretions. Nevertheless, occasional flushing with nonionic solvent is recommended for trouble-free operation. Fouling of rods can be detected by checking either the detector input with a DVM or the buffer output with a VOM. The potential should be approximately $5 \mathrm{~V}$ when the cage is empty.

\section{DEMONSTRATION}

In order to demonstrate the value of this device for measuring activity, we conducted a simple experiment in which rats' activity was measured by the activity monitor described here and directly by a human observer. It was assumed that the correlation between these two measures would be positive.

Eight satiated male rats were placed in a Skinner box for a 15-min test. An observer (R.M.T.) sat about $6 \mathrm{ft}$ from the apparatus; the room lights were off. At the onset of the session, the houselight was illuminated. Every minute thereafter, the computer initiated a 20-sec "trial" during which it recorded activity inputs from both the electronic detector and the observer. The observer operated two push buttons throughout the session: One was closed whenever the animal was active (i.e., body and feet in motion); the other button was pushed while the animal was rearing on its hind legs. The activity device also monitored activity throughout the session, but inputs from the device and from the observer were registered only during the 20 -sec trial. The data read by a computer, therefore, were activity counts (from the activity monitor), cumulative seconds spent locomoting, and cumulative seconds rearing (from the observer).

Rats rarely walk while rearing, and thus cumulative time spent rearing should not be highly correlated with activity as measured by the monitor. The Pearson correlation coefficient for activity counts and cumulative rearing was -.11 . Cumulative locomotion time and the activity counts, however, were related: $r=.69$. The coefficient could have been higher, but it was rather difficult for the observer to track locomotion accurately because animals started and stopped unpredictably and at varying speeds. Also, on occasion the subjects affected the activity monitor without actually making gross body movements that were detected by the observer (e.g., during grooming). Nevertheless, the relationship is sufficiently strong to warrant confidence in the device as an activity monitor.

\section{REFERENCES}

Aкака, W. H., \& Houck, B. A. (1980). The use of an ultrasonic monitor for recording locomotor activity. Behavior Research Methods \& Instrumentation, 12, 514-516.

BЕАсн, G. (1970). A sensitive activity transducer. Journal of the Experimental Analysis of Behavior, 14, 237-239.

CAM PBELl, B. A. (1954). Design and reliability of a new activityrecording device. Journal of Comparative and Physiological Psychology, 47, 90-92.

Campbell, B. A., \& Teghtsoonian, R. (1958). Electrical and behavioral effects of different types of shock stimuli on the rat. 
Journal of Comparative and Physiological Psychology, 51, 185-192.

Cunningham, C., Crowell, C. R., Eaton, N. K., \& Brown, J. S. (1973). A digital system for recording startle responses in small animals. Behavior Research Methods \& Instrumentation. 5, 1-3.

Davis, S. D. (1970). A continuous activity monitor for small caged animals. Physiology \& Behavior, 5, 953-954.

Hoffman, H. S., \& Fleshle R, M. (1964). An apparatus for the measurement of the startle-response in the rat. American Journal of Psychology, 77, 307-308.

Morgret, M. K., \& AlbeE, P. R. (1974). An automated openfield apparatus utilizing an improved resistance detection circuit. Behavior Research Methods \& Instrumentation, 6, 327-328.

Porter, J. J., Hudy, J. J., \& Furber, A. M. (1979). A pressureactuated open-field apparatus for rodents. Behavior Research Methods \& Instrumentation, 11, 59-60.
Tapp, J. T., Zimmerman, R. S., \& D'Encarnacao, P. S. (1968). Intercorrelational analysis of some common measures of rat activity. Physiological Reports, 23, 1047-1050.

Treichle R, F. R., \& Hall, J. F. (1962). The relationship between deprivation weight loss and several measures of activity. Journal of Physiological and Comparative Psychology, 55, 346-349.

VAN-Toller, C., \& DE SA, A. (1968). A continuous recording activity cage. Journal of the Experimental Analysis of Behavior, $11,65-67$.

Zeier, H., \& Tschannen, G. (1968). A new device for measuring general locomotor activity in instrumental conditioning situations. Journal of the Experimental Analysis of Behavior, 11, 63-64.

(Manuscript received January 20, 1984; revision accepted for publication March 30,1984 .) 